\title{
JOURNAL.RU
}

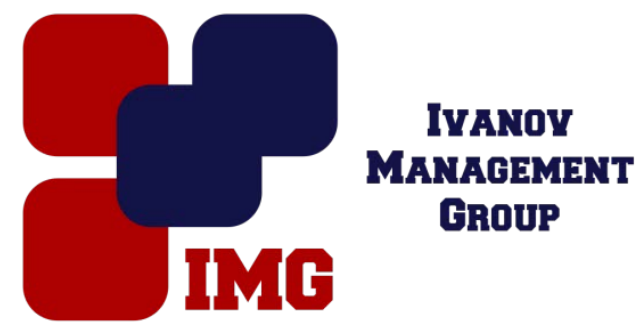

Суиндыкова Э.А., Пищин О.Н. Астраханский Государственный Технический Университет Астрахань, Россия

doi: 10.18411/lj-30-04-2017-3-05

idsp 000001:lj-30-04-2017-3-05

\section{Исследование эффективности систем мониторинга при управлении сетью передачи данных}

\section{Аннотация}

Статья посвящена сравнительному анализу существующих систем мониторинга. Выявлена общая архитектура данных систем и рассмотрен метод оптимизации ресурсов.

Ключевые слова: сеть передачи данных, сетевой трафик, протокол SNMP, распределенный мониторинг, подход «менеджер-агент».

Современные предприятия в соответствии со своими масштабами, как правило, имеют собственные локальные сети, включающие в себя серверы, точки доступа, и другое соответствующее сетевое оборудование. На крупных предприятиях очень часто сеть имеет распределенный характер. Чтобы сохранять локальную сеть в работоспособном состоянии, необходим постоянный контроль её функционирования. Процесс контроля обычно делят на два этапа: опрос параметров и анализ.На первом этапе выполняется более простая операция - процедура сбора первичных данных о работоспособности сети и устройствах, подключенных к ней: программных и аппаратных характеристик рабочих станций, работоспособность сетевых устройств и оконечного оборудования, сетевой трафик. Далее выполняется этап анализа, под которым понимается более сложный и интеллектуальный процесс осмысления собранной информации и выработки предположений о возможных причинах замедленной или ненадежной работы сети. Задачи опроса решаются с помощью системцентрализованного мониторинга и управления сетью передачи данных 
(СПД). Существует достаточно большое количество программного обеспечения, которые предназначены для анализа и наблюдения за сетевым трафиком. Каждая программа обладает своими достоинствами и недостатками.

В условиях современной конкуренции, сложно найти решение для управления, которое может подойти конкретной ситуации. Поэтому перед предприятием, желающем развернуть систему мониторинга стоит многогранная задача выбора подходящей системы.

На практике к платформам системы мониторинга предъявляются следующие основные требования [1]: масштабируемость, поддержка распределенной архитектуры «клиент-сервер» открытость, которая позволит управлять оборудованием различных производителей.

В данной статье рассмотрены следующие системы мониторинга:NagiosCore, Zabbix, Argus,IPHostNetworkMonitor, NetMRI.Результаты сравнительного анализа систем сведены в таблицу 1.

Сравнительный анализ систем мониторинга

Таблиияа 1

\begin{tabular}{|l|c|c|c|c|c|c|c|c|c|}
\hline \multirow{2}{*}{ Система мониторинга } & \multicolumn{7}{|c|}{ Параметры } \\
\cline { 2 - 11 } & 1 & 2 & 3 & 4 & 5 & 6 & 7 & 8 & 9 \\
\hline Argus & + & - & - & - & + & + & + & + & + \\
\hline Intellipool Network Monitor & + & - & - & + & - & + & + & - & + \\
\hline IPHost Network Monitor & + & + & - & + & - & + & - & + & - \\
\hline NetMRI & - & + & - & + & - & + & + & + & + \\
\hline Zabbix & + & + & + & + & + & + & + & + & + \\
\hline Nagios & - & + & - & + & + & - & + & + & + \\
\hline
\end{tabular}

Сравнение систем мониторинга происходило по следующим параметрам:

1. Формирование отчетов SLA (ServiceLevelAgreement) согласно требованиям.B SLA описываются условия предоставления услуг (сервисов), устанавливается перечень таких услуг, а также правила, по которым заказчик будет пользоваться этими сервисами [4].

2. Формирование трендов. Выявление основных тенденций динамики показателей качества работы телекоммуникационной сети.

3. Прогнозирование трендов. Прогнозирование изменения динамики показателей качества работы телекоммуникационной сети.

4. Анализ топологии сети.Сбор информации об элементах сети.

5. Использование агентной модели мониторинга.Наличие устройств, осуществляющих сбор и передачу информации о работе сети.

6. Поддержка протокола SMNP. Использование протокола SMNP для обмена информацией о состояниях объектов наблюдения в режиме реального времени. 
7. Протоколирование событий.Формирование подробных записей о состоянии элементов сети.

8. Датчики внештатных ситуаций.Наличие устройств для оповещения о возникновении критических ситуаций, негативной тенденции изменения показателей качества работы телекоммуникационной сети.

9. Распределенный мониторинг.Мониторинг сигнального обмена на предмет соответствия работы оборудования определенным спецификациям протоколов.

Проведенный анализ показал, что рассмотренные системы мониторинга схожи в выполняемых функциях. Однако каждая из них, нем не менее уникальна и обладает определёнными недостатками. Например, во всех системах не реализована возможность прогнозирования трендов. Обобщив предлагаемые решения, можно представить общую схему распределенной системы мониторинга. (Рисунок 1).

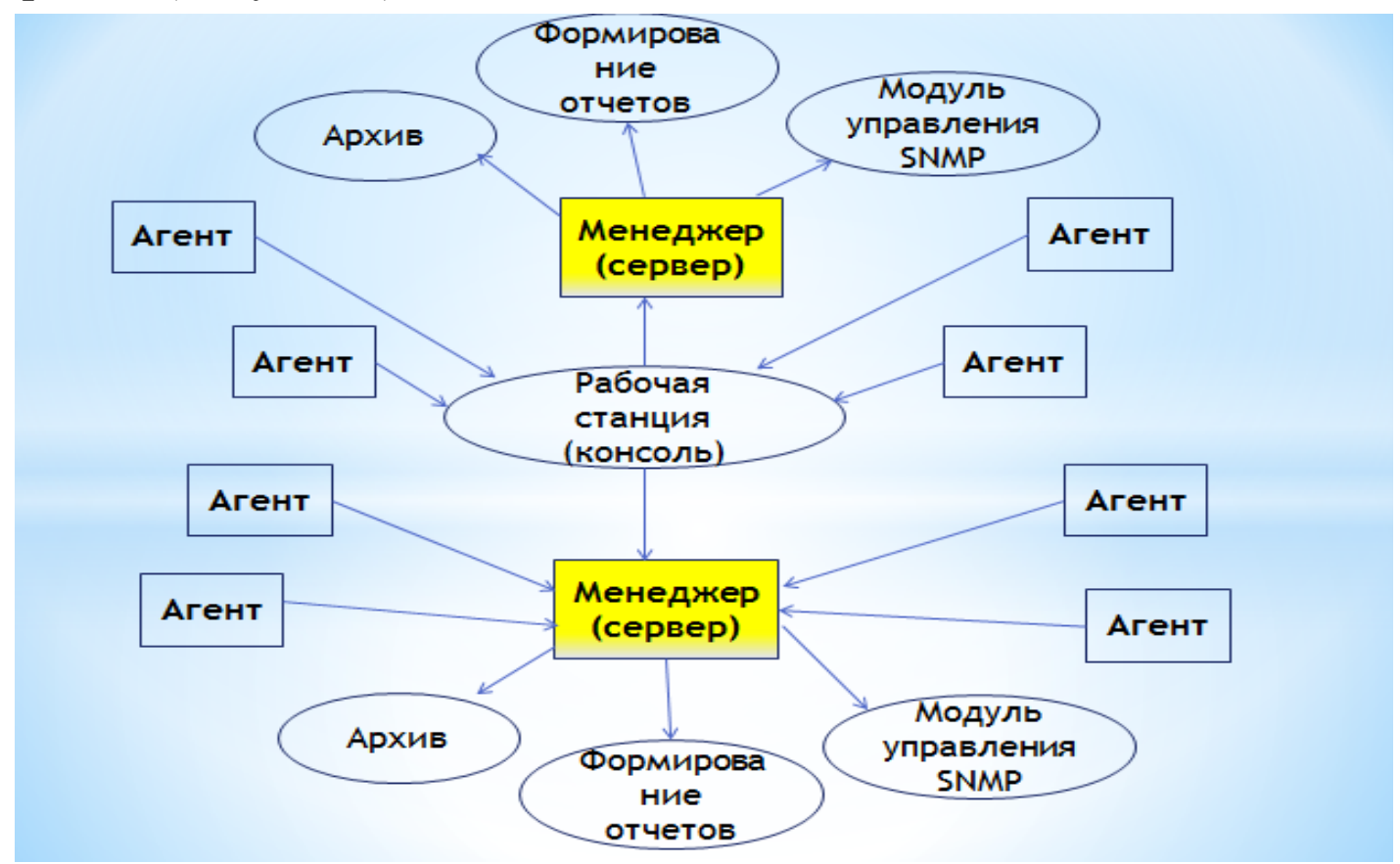

Рисунок 1. Схема распределенной системы мониторинга

Каждый агент собирает данные и управляет определенным элементом сети. Менеджер, также называемые серверами системы мониторинга, собирают данные от своих агентов, обобщают их и хранят в базах данных. Операторы, работающие за рабочими станциями, могут соединиться с любым из менеджеров и с помощью графического интерфейса просмотреть данные об управляемой сети, а так же выдать новые директивы по управлению сетью и ее элементами. 
Модуль SNMP необходим для сбора информации с агентов и взаимодействия с системами управления. Архив служит для хранения упорядочивания статистической информации. Консоль реализует управление системой.

Актуальной задачей является разработка таких систем мониторинга, которые позволили бы адаптироваться к различным размерам сетей и оптимизировать свободные ресурсы. Дополнительным требованием к такому методу является возможность не использовать ресурсы, которые уже задействованы в данный момент времени другими приложениями.

Для повышения вероятности сохранения актуальной информации необходимо уменьшение времени работы системы при неизменном количестве ресурсов. В общем виде время работы системы может быть записано в виде[3, c.32]:

$T=\sum_{1}^{n} t_{i}$, где $t_{i}-$ время необходимое для обработки і-ого устройства.

$t_{i}$ можно представить в следующем виде:

$t_{i}=t_{s i}+t_{w i}+t_{f i}$, где

$t_{s i}$ - время, необходимое для формирования и отправки запросов

$t_{w i}$ - время ожидания ответа

$t_{f i}$ - время обработки полученных данных

Обозначим за $t_{r i}$ время использования ресурсов. Тогда:

$$
t_{r i}=t_{s i}+t_{f i}
$$

Соответственно, время, необходимое для окончания работы системы можно представить в виде

$$
T=\sum_{1}^{n} t_{r i}+\sum_{1}^{n} t_{w i}
$$

Уменьшить первую составляющую при использовании стандартных аппаратных средств достаточно сложно. Вторую же составляющую - время ожидания ответа, в идеальном случае можно свести к нулю. Таким образом, можно говорить об оптимизации ресурсов по времени.

Исходя из вышесказанного, можно сделать вывод, что при минимизации времени ожидания данных можно получить выигрыш во времени обработки всех устройств, при условии недопущения перегрузок аппаратного обеспечения сервера. То есть в момент ожидания данных от одного устройства обрабатывать данные, полученные от другого [5]. 
В статье были рассмотрены наиболее популярные системы мониторинга. Исходя из полученных данных, можно сделать вывод, что многие системы являются схожими по своим функциям. Однако, большинство из перечисленных систем не способны прогнозировать состояние сети, то есть не имеют возможность предсказывать изменения основных показателей сети.

Синтезирована общая схема сети мониторинга и управления. Рассмотренные системы мониторинга основаны на использовании подхода «менеджер-агент».

Эффективность функционирования систем мониторинга определяется вероятностью сохранения актуальности данных на момент их использования.Для сохранения актуальности данных о состоянии сети, можно минимизировать время ожидания данных, таким образом, выиграв во времени обработки других устройств.

\footnotetext{
$* * *$

1. Баращ Л., Мониторинг трафика в сетях с коммутацией пакетов // Компьютерное обозрение. 2012. № 37 (654). С.20-25

2. Ahmed D.Cora, Nagios Based Enhanced IT Management System //International Journal of EngineeringScience and Technology. March 2012. № 3. P.1

3. Сторожук Д.О., Методы и алгоритмы для систем мониторинга локальных сетей // Автореферат диссертации. М., 2008. С. 31-32

4. SLA для начинающих http://www.inframanager.ru/survive_guide/sla_for_beginners/

5. Костогрызов А.И., Методическое руководство по оценке качества функционирования информационных систем // М. 2004. 352 с.
} 